\title{
Plattform III „Kunst mit Füßen treten“ Eine überdimensional große Filzaktion
}

Ina Schlüter-Zech, Projektleiterin Plattform III

In Kooperation mit Frauengruppen der Kirchengemeinden Johanneskirche und Kreuzkirche, jenseits und diesseits der Bahn, und PassantInnen der Innenstadt, August bis September 2005

Als ich gefragt wurde, in einem Modellprojekt, dessen Ziel zeitgenössische Kunstvermittlung von Kunstschulen ist, eine Plattform zu gestalten, habe ich mich gefreut. Unsere Metapher lautete „Tunnelblick“. Ziel sollte sein, mit Menschen zu arbeiten, die nicht aus dem Kunstschulbereich kommen.

Überlegung 1: Ein Tunnel verbindet Orte und Menschen, der Tunnelblick lässt eine eingeschränkte Sichtweise zu, eröffnet nach dem Durchgang einen neuen Blick, verändert Blickwinkel, so wie es auch die Kunst schafft.

Überlegung 2: Welche Teilnehmerlnnen? Welche Gruppe von Menschen gibt es jeweils auf beiden Seiten des Tunnels? An welchem Ort kann eine Aktion stattfinden?

Ich entschied mich schließlich für zwei Kirchengemeinden, die dies- und jenseits des Tunnels angesiedelt sind. Der Ort sollte einer großen Öffentlichkeit zugänglich sein, und es sollte Kunst als Nebenprodukt (also rein zufällig), als Beiwerk entstehen. Eine Intervention, bei der die Aktion die Kunst und nicht das Produkt zur Kunst wird. Es sollte sich um etwas handeln, was diese beiden Gruppen immer wieder an diese Aktion erinnert. Ich forderte die Frauengruppen in den jeweiligen Kirchengemeinden auf, mit mir diese Plattform zu erarbeiten.

Aufgabe: Es soll eine Kunstperformance auf dem Marktplatz entstehen, an der alle PassantInnen beteiligt werden, mit oder ohne aktive Einflussnahme.

Idee: Wolle wird auf dem Marktplatz verteilt, von PassantInnen und den beteiligten Personen mit Wasser und Seife besprüht und dann von darüber laufenden Personen zu Filz verdichtet.

Ich habe bewusst die Art des Filzens gewählt, um auf die Tunnelmetapher wieder einzugehen. Wenn man einen Tunnel verlässt, begegnet man anderen Menschen, sieht Neues; mit der Zeit verfilzen sich auch Kulturen und Andersartigkeiten. Den beiden ausgewählten Gruppen erläuterte ich meine Idee und forderte sie auf, als meine Mitstreiterlnnen zu fungieren. Um so eine große Performance durchzuführen, brauchte ich Menschen, die mir auf dem Marktplatz halfen. Ich bot an, 
in zwei Aktionen das Prinzip des Filzens zu vermitteln, um danach die Aktion starten zu können. Das dabei entstehende Produkt sollte dann im Wechsel beiden Gemeinden zur Verfügung stehen: Einmal im Jahr werden sich diese beiden Gruppen wieder treffen, um die Arbeit auszutauschen. In einem gemeinsamen Gespräch entschieden sich die beiden Gruppen, einen Wandteppich mit zwei unterschiedlichen Seiten zu filzen. Wir trafen uns, um die Wolle für den Teppich auszulegen. Jetzt stand der Aktion auf dem Marktplatz, der sich auf der anderen Seite des Tunnels befindet, nichts mehr entgegen. Ein Termin wurde gefunden, die Genehmigungen von der Stadt Lingen erteilt, ein großer Aufruf in der Zeitung gestartet, und los ging es. Unter einem Zeltdach wurde der Wandteppich, der sich noch in einem losen Zustand befand, gelegt und die Frauen fingen an, ihn mit Wasser und Seife zu filzen. Eine Zuschauermenge bildete sich und wurde aufgefordert, Wolle auf dem Marktplatz zu verteilen, egal wo - nur ein Wollfaden sollte als Verbindung zu anderen Arbeiten mit eingebunden werden. Ein reges Treiben entstand, selbst Unbeteiligte mussten über die Arbeiten laufen. So verfilzte sich die Wolle unter anderem durch eine Hochzeitsgesellschaft, einen Rollstuhlfahrer und viele PassantInnen. Ständig kamen neue hinzu. Meine beiden Frauengruppen sprachen Menschen an, arbeiten an ihrem Teppich, führten rege Diskussionen über Kunst und anderen Unsinn. Nach fünf Stunden wurde die Aktion erfolgreich beendet.

Nachlese: Ein Treffen der beiden Gruppen und eine Nachlese standen auf dem Programm; ich erarbeitete dazu einen Fragebogen, den alle Teilnehmerinnen gemeinsam in einer Gruppendiskussion beantworteten. Hier das Ergebnis:

Frage 1: Wie war die Reaktion der PassantInnen zur Anfrage einer Beteiligung am Projekt auf dem Marktplatz?

Wurde positiv aufgenommen, mal was ganz anderes.

Frage 2: Haben Sie nach dem Warum gefragt?

Nein, in keiner Gruppe.

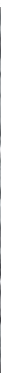

Plattform III, Fotografien: Reinhard Prüllage 
Frage 3: Denken Sie, dass es eine zeitgenössische Vermittlung von Kunst war? Nein, eher revolutionär.

Frage 4: War es ein Kunstprojekt?

Der Wandteppich war Handarbeit, also Vermittlung einer Technik. Aber auf dem Marktplatz das Filzen in Aktion, wo auch Unbeteiligte sich als Akteurlnnen unbewusst befanden, das war eine Kunstperformance.

Frage 5: Was hatten Sie für Vorstellungen?

Keine großen; Ergebnis übertraf Erwartungen; von sich selbst und dem Ergebnis überrascht.

Frage 6: Würden Sie öfter an solchen Aktionen, die mit Ihrer normalen Tätigkeit und Gruppenarbeit wenig zu tun haben, teilnehmen?

Ja, warum nicht.

Frage 7: Würden Sie sich um solche Projekte bemühen?

Nein, auf solche Ideen kommen wir nicht. Aber es ist toll, mal etwas ganz anderes zu machen, auch als Gruppe. Die verschiedenen Orte waren auch ideal, jedesmal die Einstellung auf einen anderen Ort, da wurde einem bewusst, wie viel Einfluss das doch hat.

\section{Mein persönliches Fazit zum Modellprojekt}

Durch die verschiedenen Plattformen, die wir in Eigenregie ausgeführt haben, ist es uns gelungen, auf ein großes Erfahrungsspektrum zurückzugreifen, viele verschiedene Gruppen einzubeziehen. Obwohl nur unsere Metapher uns zusammenhielt, ist der kausale Zusammenhang in der Ausstellung allen Beteiligten klar geworden. Kritik seitens der wissenschaftlichen Begleitung und der Geschäftsstelle des Landesverbandes zu meinem Projekt, es handele sich in ihren Augen nur um das Erlernen einer alten Technik, haben dazu geführt, dass ich durch viele Überlegungen, was meine Grundidee angeht, nach wie vor überzeugt war und mich bestärkt fühlte, mit meiner geplanten Projektstruktur zeitgenössische Kunstvermittlung gestalten zu können. Meinen eigenen Arbeiten habe ich damit mehr Respekt gezollt. Denn, wenn ich etwas aus dem Kunststudium gelernt habe, dann: Kunst soll Fragen und Widerstand aufrufen. 


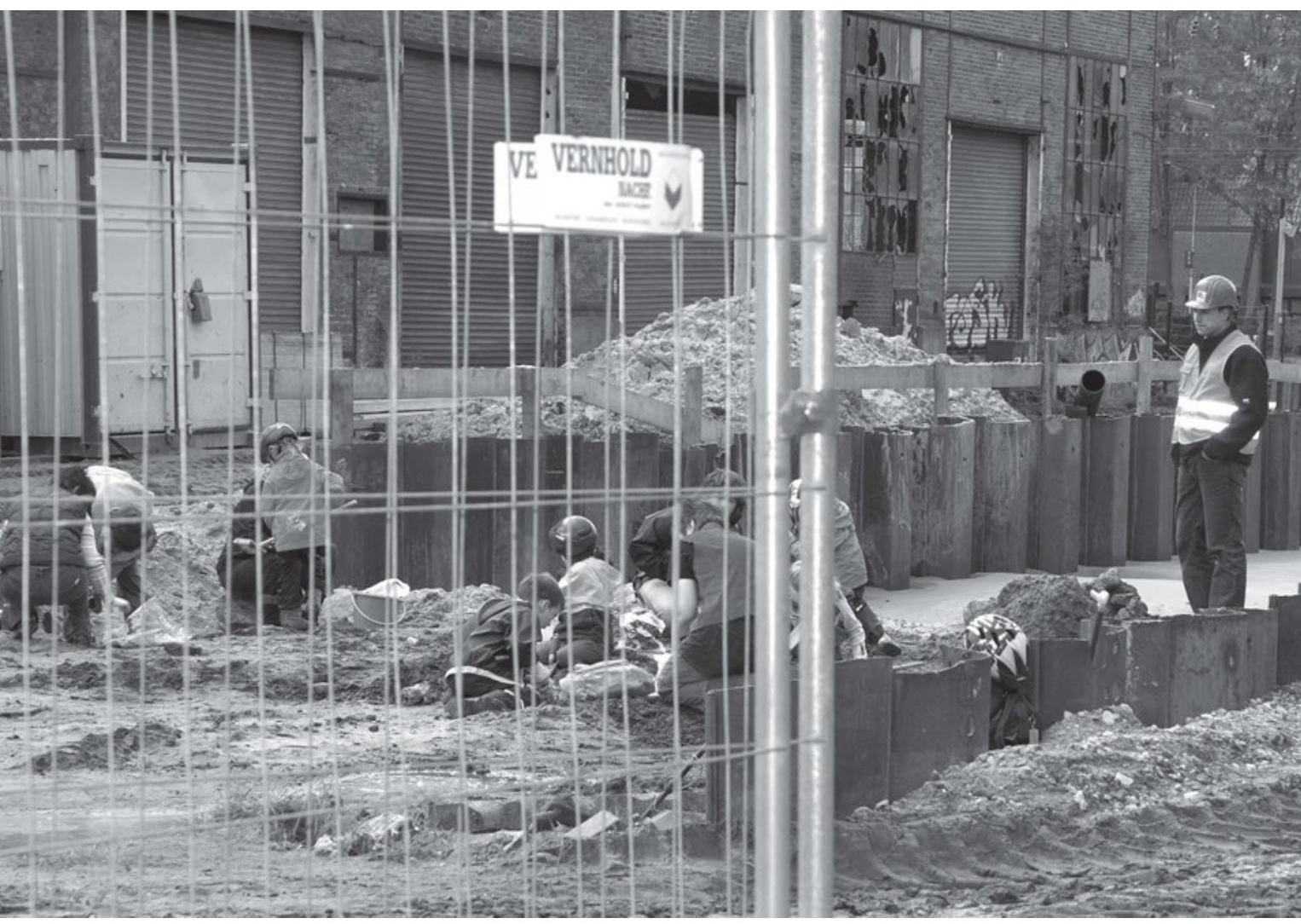

